\title{
THE DIAGNOSIS AND TREATMENT OF CARDIAC PAIN.
}

\author{
By GEOFFREY BOURNE, M.D., F.R.C.P.
}

(Assistant Physician, Officer in charge Cardiology Dept., St. Bartholomew's Hospital; Physician, King George Hospital, Ilford.)

The most necessary factor in treatment is accurate diagnosis and there is no subject where this is more true than in that of cardiac pain. The chief conditions in which cardiac pain occurs are,

I. Coronary Thrombosis,

2. Angina of Effort,

3. Spasmodic Angina, and

4. Angina Innocens.

\section{CORONARY THROMBOSIS.}

Coronary thrombosis is now well recognized as a clinical entity, and its symptoms and signs are understandable in the light of the pathological changes which are found by post-mortem examination.

\section{Pathology and Symptoms.}

The sudden blocking of the artery produces an abrupt anæmia of a considerable mass of living heart muscle. This produces pain. Certain changes take place in the infarcted area, necrosis, fibrosis, and to a certain extent recovery which occurs by virtue of the presence of some collateral circulation at the periphery of the infarct. As these changes proceed, the pain slowly dies down, and has generally completely disappeared by the end of the week. The amount of pain varies greatly from case to case, and in some cases is comparatively mild.

The characteristics of the process are the sudden onset, the fact that the pain is at its greatest at first, and that it slowly subsides during a period of days. The pain may be persistent during that time, but it is often remittent or intermittent. The associated pericarditis is painless. In some cases there is a relation between pain and respiration, but this is generally not greatly complained of and is rarely the cause of difficulty in diagnosis. The pain is generally substernal, and may or may not radiate to the arms. It tends to be more definitely localized to a spot than do the pains of angina of effort or of spasmodic angina. It may be referred to the upper abdomen. The pain of coronary thrombosis is not relieved by amyl nitrite.

As a result of the interference produced in the coronary circulation, an attack of coronary thrombosis frequently is the starting point for the angina of effort syndrome. The symptoms of this appear after recovery from the infarction, when on exercise some of the surrounding muscle becomes anæmic and pain results.

The sudden local disease and death of a fairly large area of ventricular muscle also produces an abrupt limitation of the cardiac response to exercise, so that a patient previously well states that since his attack of pain he has been markedly short of breath on exertion, and has felt weak. 
The characteristic physical signs of coronary thrombosis are due to three causes, necrosis of and hæmorrhage into the heart muscle, myocardial disability and pericarditis.

The myocardial necrosis and hæmorrhage give rise to fever and leucocytosis. These are present in most cases if looked for, but the fever may be easily missed if a rectal temperature reading is not taken, owing to the presence of shock.

The myocardial weakness is shewn by the feebleness of the cardiac impulse and the cardiac dilatation (which are almost invariably present), the poor quality of the first sound, the tic-tac rhythm, and by the definite lowering of the systolic blood pressure.

The pericarditis may or may not produce a friction rub. distant and may be transient. It is usually not heard before the third day after the thrombosis, and may not appear until the fifth or sixth.

The electro-cardiographic changes (see page I23) vary according to the site of the lesion. The universally applicable rule is that after a coronary thrombosis the shape of the ventricular complex, whatever form this may take, changes abruptly from the normal within the first one to three days after the thrombosis has occurred, and subsequently rapidly varies from day to day and from week to week. The period over which obvious change continues to occur is roughly six weeks, and the maximum amount of change occurs during the first ten days. It is advisable to take the first tracing within the first three days after the onset of the symptoms, and a subsequent one after ten days and a third may be taker during convalescence. Apart from the rapidity of rate of change of shape, the electrocardiographic curve of coronary thrombosis often presents certain definite abnormalities within the first few days, such as Pardee's sign (page I25), which are pathognomonic of the condition.

\section{Treatment.}

The treatment of coronary thrombosis falls into two parts, the treatment of the emergency and the treatment of the damaged heart.

Treatment of the Emergency. Pain is the outstanding feature and demands instant relief. Amyl nitrite and nitroglycerine are often given as a preliminary measure, but they have no effect on the pain. This is one of the points which help to differentiate the condition from spasmodic angina. Torphia is the only reliable drug in the treatment of the pain of coronary thrombosis, and the writer has never seen harm result from its use. The dose must be sufficient to control the pain. The generally effective dose is gr. $\frac{1}{4}$ four hourly, but gr. $\frac{1}{2}$ four hourly is necessary in some very severe cases. If pulmonary œdema is also present atropine sulphate gr. I/ Ioo can be given simultaneously. On the second and o subsequent days the amount of morphia can be reduced, and as a rule by the $N$ fourth day the drug is no longer necessary. A subsidiary effect of the morphia is to remove the great restlessness which is often present, and which throws an added strain upon the damaged heart.

Anorexia is often present, though generally to a slight degree only. It $\stackrel{\Phi}{\stackrel{D}{\odot}}$ is increased if pulmonary odema has resulted from the left-sided heart failure. Oxygen in cases of heart failure is often ineffective, for the cyanosis results from general slowing of the circulation rate. This not only gives more time for the tissues to remove oxygen from the hæmoglobin, thus producing cyanosis, but it 
also gives more time for the pulmonary capillary blood to receive its full quota of oxygen. Thus an increased oxygen tension in the inhaled air can add nothing to the oxygen capacity of the hæmoglobin of the pulmonary blood. If, however, pulmonary œdema is present, a respiratory anoxæmia, in addition to a circulatory anoxæmia, may be present. In these circumstances an increased oxygen tension in the inspired air will help to force oxygen through the fluid layer lining the pulmonary alveolar walls. Oxygen is thus indicated in coronary thrombosis if râles are present at the pulmonary bases. The funnel method is useless; oxygen must be given by mask, tent, or in a good stream by nasal catheter.

Rest is essential for the patient with a coronary thrombosis, and this can only be obtained by adequate nursing. The patient must be confined to bed, and should rest as flat as is comfortable. All unnecessary exertion in washing, feeding and in the use of the bed-pan must be avoided.

The commonest period for death to occur, and therefore the most dangerous, is from the sixth to the twelfth day. After that the amount of exertion allowed, in bed, can gradually be increased. The safe general rule is that rest in bed should be continued for at least six weeks.

There are two exceptions to the above rule. If the thrombosis has been a small one - often a difficult matter to decide-, or if the patient is an old man or woman, the period of strict rest in bed can be curtailed. It is generally wise, however, even in these circumstances to insist that the patient remain strictly in bed for twelve days at least. In every case where the patient is allowed up within six weeks a definite risk is being taken. Hypostatic pneumonia, urinary infection, and the loss of muscular tone and of general health, which so quickly overtake old persons confined to bed for a long period, are the dangers against which the risk of allowing them up too soon have to be balanced.

Three considerations control the choice of diet for patients suffering from a coronary thrombosis. The diet must not be bulky, it must contain plenty of sugar, and it must be palatable. An excess of fluids and farinaceous foods likely to cause gastric distension must be avoided. Dry concentrated solids are advisable if the patient can take them. Sugar can be given as glucose or cane sugar.

The bowels must be kept open, but violent purgation is not advisable. The habits of each patient must be considered individually.

Treatment of the Damaged Heart. The two permanent pathological results of coronary thrombosis are the death of part of the infarcted area, and some degree of local fibrosis. Neither of these necessarily produces permanent cardiac disability, for hypertrophy of the remaining normal muscle fibres can occur, and a scar is an inert structure. But a fully compensatory hypertrophy can only occur if the injury has been sufficiently small, and if the remaining muscle fibres are uninfluenced by toxæmia or anoxæmia. It is thus theoretically possible for a complete recovery to follow a coronary thrombosis, and this result does occur in some patients.

After the period of six to eight weeks in bed, a slow convalescence is carefully supervised, more and more exertion being slowly allowed. The patient should be allowed gradually to undertake increased exertion to an extent which produces neither dyspnœea or unnecessary fatigue. This degree of exertion may in favourable cases reach the normal figure. 
In patients with more severe myocardial damage, or with hyperpiesis or some other complicating lesion, although the same rule can be followed; it will be found that progress is slower and the amount of exercise tolerated is proportionately less. When patients who have suffered from a coronary thrombosis have recovered to their full extent, they should be examined carefully and any other mild disability should be rectified. Attention should particularly be directed to the possible presence of dental sepsis, urinary infection, chronic cholecystitis and mild diabetes.

In diabetics with coronary thrombosis the sugar in the diet should not be cut down during the acute stage. Insulin in doses sufficient to control the glycosuria should be given twice daily. It is safer to give too little rather than too much insulin to such patients.

\section{ANGINA OF EPFORT.}

\section{Nature and Symptoms.}

This form of cardiac pain is the most characteristic of a structural coronary lesion. It is produced either by atheroma of the coronary mouth, or of part of the coronary stem, or by involvement of the coronary mouth in a syphilitic aortitis. Its characteristics are a sense of oppression, subsequently increasing to pain, and only occurring on exertion. In all cases there is a definite level of exertion at which the symptoms arise. Below this level there is no discomfort, and above it in proportion to the severity of the exertion the symptoms increase. The rate of development and the severity of the exertion determine the rapidity of onset and the degree of discomfort and pain. Conversely, rest inhibits the development of symptoms and relieves them when they are present.

The symptoms are due to myocardial anoxæmia produced by the failure of the diseased coronary vessel to transmit more than a fixed quantity of blood. The pain is generally substernal and may radiate to one or both arms. In some cases it is felt primarily in the wrists or arms and secondarily in the chest.

The pain often comes on more easily in cold weather, or after a meal, and may thus give rise to a mistaken diagnosis of dyspepsia ; but the relation to exercise is still constant.

The three commonest conditions with which the symptom is associated are hyperpiesis, a previous coronary thrombosis, and syphilitic aortitis. In some cases, however, there is no clinical evidence of any of these diseases.

\section{Prognosis.}

Although the symptoms of angina of effort always indicate a coronary lesion, the extent of this, and the severity of the associated myocarditis, vary greatly.

An exact evaluation of the severity of the disease is a matter for careful investigation in each case, but certain simple rules are generally applicable. Briefly, they are as follows:

If the patient is able, during periods of remission of symptoms, to walk well, climb stairs and hills, and undergo reasonable exertion, the myocardium is reasonably sound. 
If the pain is not so great as to prevent all exercise, and if during this exercise dyspnœea is not complained of, the myocardial condition is fairly good.

If marked enlargement of the heart, hyperpiesis, or some subsidiary condition such as diabetes, are present, the outlook is more serious. In angina of effort due to atheroma the heart is generally more or less enlarged; in angina of effort due to syphilitic aortitis the heart is normal in size unless aortic incompetence is present.

If pulsus alternans, Cheyne-Stokes breathing, attacks of cardiac asthma, are found, the outlook is bad.

A second point to determine carefully is the degree of sensitivity of the nervous system; for although angina of effort is a symptom of organic disease, the amount of coronary obstruction sufficient to produce symptoms varies greatly according to the reactivity of the individual nervous system of the patient.

\section{Treatment.}

There is no disease in which a full examination from a general point of view is more essential than in angina of effort. A full cardiological examination is of course necessary but from the point of view of successful treatment a far wider view-point is desirable. A discussion regarding treatment therefore divides itself into two sections, that dealing with the cardiac state and that dealing with associated conditions.

Reat in bed is not necessary unless signs of severe myocardial failure are present. The patient should be allowed, and indeed encouraged, to take that amount of exercise which does not produce symptoms of oppression, pain or undue dyspnœa.

A most important means of relieving the heart of unnecessary work in adipose individuals is weight reduction. Two stone in weight produces an equal handicap whether it is in the form of lead weights in the pockets, or in the shape of adipose tissue in the thorax, peritoneum or buttocks. The reduction in weight should be brought about gradually and by dietetic means, potatoes and other starchy foods, sugar, pastry, fat, cream and excess of butter being avoided. Rye Vita or other such preparations can be substituted for bread.

Rest for half an hour to an hour is advisable in these patients after the chief meals of the day. Alcohol in small quantities with meals is not contraindicated, tobacco is best avoided.

Drug treatment is often helpful. Nitroglycerin tablets (gr. I/ 200 to gr. I/50) will relieve the pain if it is severe, but as its onset is generally gradual they are less necessary for this purpose than they are in spasmodic angina. Their chief function is that if it is known that pain is likely to be incurred at a particular time, or as a result of some definite piece of exertion, they can be taken safely immediately previous to this. They should be crushed in the mouth and held there for several minutes before being swallowed in order to obtain a full effect.

Luminal gr. $\frac{1}{4}$ to gr. $\frac{1}{2}$ t.d.s. p.c. often helps these patients considerably.

A group of drugs which in the writer's experience seems to be of use in certain cases is the theobromine group. Theobromine, theobromine sodium salicylate, theophyllin, euphyllin, are all worthy of a trial in a severe and protracted case. Each should be tried separately for a period of a month, and occasionally a marked improvement seems to occur. They are given continuously three times a day. 
Syphilitic aortitis as a cause of angina of effort needs full and determined treatment. The results in cases where the Wassermann reaction has been rendered and kept negative are very satisfactory; the pain and dyspnoea disappear and the patient returns to perfect health. A preliminary course of potassium iodide and mercury is given, the iodide being increased from gr. $\mathrm{xv}$ to gr. $\mathrm{xx}, \mathrm{xxx}$ or $\mathrm{xl}$ four times a day. After two months, a course of bismuth, or of intravenous neosalvarsan is given, the starting dose of the latter being 0.I5, and the top dose 0.6 grammes, 3.5 to 4.0 grammes being given in all. This course must be repeated at intervals of 6 to 9 months for an indefinite number of times until the Wassermann reaction becomes and remains negative.

It is in the non-syphilitic cases of angina of effort that treatment is more disappointing, and needs to take particular account of certain associated abnormalities. Some of those which have been met with by the writer include diabetes, anæmia, dyspepsia and hypothyroidism. In each of these careful treatment has often removed or alleviated the anginal pain. The necessity for treating diabetics suffering from coronary disease by a full carbohydrate diet, balanced by insulin, has been stressed above. Anæmia of whatever kind, primary or secondary, must be watched for and treated; for it is obvious that a decrease in the hæmoglobin will accentuate the effects of an already existing coronary stenosis. Quite apart from the post-prandial exacerbation of symptoms common in angina of effort, a true dyspepsia often exists, and the treatment of it by the method appropriate to the case is often followed by a great improvement in the cardiac symptoms. Hypothyroidism may exist in a mild degree in many patients, and must always bee watched for. One of the most striking cases of the writer's in whom angina of effort disappeared under treatment, was a patient with mild myxœdema treatede with thyroid extract.

It is thus clear that a purely cardiological attitude towards patients with cardiac pain will of necessity be inadequate trom the point of view of treatment. All discoverable abnormalities must be sought, carefully considered, and if necessary put right.

\section{SPASMODIC ANGINA. \\ Pathology and Symptoms.}

The pain of spasmodic angina is sudden, severe and constricting, and is associated with a feeling of impending death. It has the usual situation and radiation of other types of organic cardiac pain. It characteristically yields to amyl nitrite inhalation.

An attack is often precipitated by exercise, exposure to cold, and emotion. The quantitative relationship to exercise so typical of angina of effort is not present, and rest does not cause the attack to subside. Once started the attack has to run its course.

Spasmodic angina is found generally in association with angina of effort, and is usually a symptom of coronary disease. Cardiac enlargement is generally present, hyperpiesis, aortic regurgitation, and syphilitic aortitis are also often found. There is in addition a hypersentivity of the nervous system, the disease being found $\stackrel{\Phi}{\oplus}$ in nervous, highly strung individuals, and commonly in those whose nervous sys- 0 tems are from the nature of their work in a condition of sustained tension or $\stackrel{0}{0}$ application. Doctors, schoolmasters and clergymen and busy business men are especially liable. 
The actual cause of the attack is obscure, but the theory that it is produced by coronary spasm is a reasonable one. The action of amyl nitrite as a vascular antispasmodic would thereby be explained.

\section{Treatment.}

The remarks made upon the treatment of angina of effort are applicable to the treatment of spasmodic angina between attacks. In addition, treatment must be directed towards the attacks of pain both prophylactically and therapeutically.

Prophylactically heavy meals must be avoided, food being dry and concentrated rather than fluid and bulky. Exposure to cold must be shunned. The patient must be shielded from violent emotional upsets. Luminal is perhaps the best prophylactic drug, but some patients seem to obtain help from one or other members of the theobromine group described under angina of effort.

For the attack the nitrites are specific, and seeing that the onset is more abrupt than is that of angina of effort amyl nitrite is better here than is nitroglycerine by mouth.

Symptomatic treatment of the pain by mechanical means may be necessary both for spasmodic angina and for angina of effort; the indications for and against this are discussed later.

\section{ANGINA INNOCENS. \\ Pathology and Symptoms.}

Angina innocens is a condition unassociated with organic disease of the heart so far as can be discovered by clinical, electrocardiographic and radiological examination. There are two main types of pain; one is persistent and one spasmodic or sudden in type. The former is the commoner. It is generally præcordial rather than central, but frequently radiates to the arms as in the case in angina of effort and spasmodic angina. It never has a truly quantitative relation to effort, but is often forgotten when this is exciting or pleasurable. The effect, however, of any unusual spell of exertion is to make the pain worse afterwards. The pain may persist for days or weeks, with considerable variation in intensity. The second type of pain is spasmodic and sudden. The commonest story is that the patient, who is often standing at the time, is suddenly siezed by a severe spasm of præcordial pain, which may radiate to the arms. Within a few seconds of the onset of the attack the patient faints. The effect of these attacks is to cause an exacerbation of the previously existing continuous type of pain described above, with which the sudden spasmodic attack is nearly always associated.

There are certain other clinical characteristics of angina innocens. It generally occurs in nervous individuals, and often in those who have suffered from nervous breakdowns and other psychoneurotic conditions. Its incidence is equal in the two sexes. Hyperæsthesia of the præcordium is very common, whereas in organic pain due to organic causes this is unusual. A history of fainting and giddiness is frequent.

On physical examination no cardiac enlargement is present, and typically there is no evidence of any endocardial or other organic lesion. The cardiac action is often irritable, and the pulse rate increases out of proportion when some stimulus, physical or psychic, is applied. Hyperæsthesia is almost invariably felt over the præcordium. Its absence is a definite point in favour of an organic lesion, in a 
case of cardiac pain. The blood-pressure mechanism is labile, the reading being normal when the patient is lying flat, and lowered on assumption of the erect posture.

It must be remembered that the presence of an organic lesion is no protection against the onset of cardiac hyperexcitability, so that occasionally the syndrome may be found in patients with definite morbus cordis. Mitral stenosis, a state where a flapping ventricular action is so common, is not infrequently associated with the symptoms of angina innocens. In these cases the presence of the cardiac pain can be completely disregarded in assessing the prognosis. Moreover, the presence of an organic lesion in no way changes the clinical picture of angina innocens which is still readily recognizable.

\section{Prognosis.}

As regards the cardiac condition the ultimate prognosis is excellent, for recovery invariably occurs. The symptoms, however, frequently remain for years and the rate of recovery is often slow.

\section{Treatment.}

The treatment is difficult, and rapid results are not obtained. The most important point is to be able to state definitely to the patient that "angina " is not present; for when this sinister label has been wrongly applied to patients with angina innocens the psychological results may be disastrous; and there is in some cases a real risk of suicide. It is also essential to make a complete examination with the idea of dealing with septic foci. Chronic tonsillitis is present in somecases and its treatment may rapidly be followed by cure. Sinusitis, dental sepsis and urinary infection may also be present. The patient șhould be encouraged to take carefully graduated exercise. Any sudden overexertion is liable to accentuate the cardiac hyperæsthesia, indeed a bout of unusual effort is not infrequently the exciting cause of the syndrome.

\section{OPERATIVE .TREATMENT OF CARDIAC PAIN.}

The first essential point to bear in mind is that the differentiation of the various types of cardiac pain is so recent that most of the work on operative, or other mechanical means of treatment of " angina " needs to be carefully reconsidered in the light of this differentiation.

For example, a case of "angina" may be reported as being alleviated by operation. There is often no exact information as to whether the case was one of angina innocens with a considerable neurotic element. In such a case any form of treatment similarly impressive in degree might have produced a beneficial effect. Further, there is generally in such lists of reported cases no attempt to differentiate the cases of spasmodic angina from angina of effort. It therefore follows in any given case in which operative treatment is considered that the decision must be made from a consideration of first principles, and without certain knowledge of previous results.

The mechanical methods available are cervical sympathectomy, alcohol injection if the sympathetic rami communicates, thyroidectomy, and X-ray treatment.

$\mathbf{X}$-ray treatment is more or less universally applicable to all cases, about an erythema dose in all is given, over an area to the left of D.I to D.5, in weekly 
doses. Some cases are definitely helped, more particularly the angina innocens group, and those with sensitive nervous systems. The possibility of suggestion being largely responsible for the improvement cannot be ruled out.

Sympathectomy is perhaps a surer method than is alcohol injection for the treatment of angina of effort or spasmodic angina, and if a surgeon is available who is sufficiently skilful and conversant with the operation, it is probably the method of choice.

\section{Contra-indications for Operation.}

Surgical treatment is not advisable if medical treatment is producing a satisfactory result. Nor is it necessary where the pain is of the angina of effort type, and of a mild degree.

It is not indicated where the pain is slight or moderate, and where it serves to restrain the activities of a patient whose myocardium is grossly diseased.

\section{Indications for Operation.}

I. The pain must be of the " organic" type. Angina innocens will inevitably remit in time without operation.

2. The myocardium must be reasonably healthy.

One test is essential before it can be assumed with certainty that the state of the heart is satisfactory, and that is the response to exercise. If the patient on his "good days" is able to walk reasonably well, and without dyspnoea, or is able to climb stairs well, or undergo some other fairly searching type of exercise, it may be assumed that, provided the pain is of the "organic" type, a good result will follow.

The electrocardiograph and the X-rays are valuable means for indicating positive abnormalities, but if they give apparently normal results, this does not necessarily indicate a healthy and safe cardiac condition. A patient of the writer's suffered from very severe angina of effort on the slightest exertion, the pain being so severe as to make his life miserable. Further investigation shewed that the electrocardiograph was normal, except for some slight left-sided preponderance; an X-ray examination of the heart shewed very little enlargement, the heartchest ratio being 0.5 . The Wassermann reaction and the basal metabolic rate were both normal. The patient was transferred to a surgical ward where he died suddenly, before operation. The autopsy shewed that the mouths of both coronaries were so stenosed that they just admitted bristles. In this case, if an exercise tolerance test had been possible dyspnœa and distress would certainly have occurred with very slight exertion.

A third essential in selecting cases of cardiac pain for operation, is to make sure that the lesion is not syphilitic. A syphilitic aortitis producing pain is amenable to specific treatment, as has been described above under angina of effort.

Thyroidectomy in some chosen cases produces a good result, but in clinics where large numbers were rapidly done it is now apparent that the results are not as good as had at one time been expected. It must further be remembered that the resulting degree of myxodema, though it can be partially relieved by thyroid extract, is not an unmixed blessing to the patient. 